Proyecciones Journal of Mathematics

Vol. 36, No 3, pp. 397-422, September 2017.

Universidad Católica del Norte

Antofagasta - Chile

\title{
The circle pattern uniformization problem
}

\author{
Armando Rodado Amaris \\ Universidad de Los Lagos, Chile \\ and \\ Gina Lusares \\ Universidad de Valparaiso, Chile \\ Received : October 2016. Accepted : May 2017
}

\begin{abstract}
The existence of an explicit and canonical cell decomposition of the moduli space of closed Riemann surfaces of genus two shows that each Riemann surface of genus two can be parametrised by a 12-tuple of real numbers which corresponds to the angle coordinates of a graph associated to the surface. This suggests a Circle Pattern Uniformization Problem that we have defined and solved for three classical Riemann surfaces of genus two. Although in general, finding the exact algebraic equations corresponding to a hyperbolic surface from angle coordinates is a hard problem, we prove that known numerical methods can be applied to find approximated equations of Riemann surfaces of genus two from their angle coordinates and graph data for a large family of Riemann surfaces of genus two.
\end{abstract}

Keyword : Uniformization Problem, Riemann Surfaces of Genus Two, Circle pattern Uniformization Problem. 


\section{Introduction}

The uniformization theorem, which states that any Riemann surface of genus greater or equal than two is the quotient $\mathbf{H} / \Lambda$ of the hyperbolic upper plane $\mathbf{H}$ by a group of Möbius transformations $\Lambda$ acting on $\mathbf{H}$, leads to the classical uniformization problem which was initially developed by Poincaré, Klein and others [7, 12, 29] . Since Riemann surfaces can be viewed also as algebraic curves, the classical unifomization asks for the alternative representation, given either the curve algebraic equation or the group $\Lambda$.

The efforts that have been made to solve the classical uniformization problem have produced several versions. Among them are the Schottky's uniformization problem and the Myberg's numerical unifomization problem. In addition, the theory have been enriched by the connection that have been established to Fuchsian ordinary differential equations, $\Theta$-series, etc.

On this paper, we propose a new branch of uniformization problem: the Circle Pattern Uniformization Problem, which brings a new more intuitive perspective to the topic, by viewing each Riemann surface as a hyperbolic polyhedra that has a cell decomposition, together with a circle pattern structure and an associated graph.

A view of hyperelliptic Riemann surfaces as graphs embedded on the two dimensional sphere with angle coordinates is suggested by a solution of the problem of finding a canonical cell decomposition of the moduli space of closed Riemann of genus two, as described on [2], and leads to a circle pattern uniformization problem that consists of determining the perspectives of a Riemann surface: A) its algebraic equation, B) its hyperbolic structure, C) its associated graph and angle coordinates, when only one of them is given.

Advances toward the solution of the circle pattern uniformization problem will enrich Riemann surfaces theory and give insight into the solution of the classical uniformization problem. We restrict our discussion to the genus two case because the canonical cell decomposition of the moduli of Riemmann surfaces of genus two $M_{2}$ have been explicitly computed $[2,25]$ and then we can build on the knowledge about the structure of $M_{2}$ to get explicit results.

We will show that numerical methods to find the algebraic equations of genus two surfaces from a surface hyperbolic structure, which have been studied by several authors $[12,29,7]$, can be applied to study the circle 
pattern uniformization problem and leads to a new methodology to find the algebraic equation of Riemann surface from its circle pattern representation.

The angle coordinates associated to a generic graph $G$ corresponding to a Riemann surface of genus two, $R$, carries all the information that is needed to construct a symmetric octagon representation of $R$. In addition, from $R$ octagon's representation one can determine the hyperbolic lengths of its dual graph's sides. Hence, we can identify the pants length and twisting parameters of $R$, and approach computationally the circle pattern uniformization problem because there are known computer programs [7] which compute good approximations for the algebraic equations of a curve from a surface's pants parameters.

To study the classical uniformization problem, Buser and Silhol [7] reduced the problem to the well known genus 1 case: Given a surface $R$ of genus two, they construct a double cover $Y$ of $R$ of genus three. By a result of Enriques, $Y$ is hyperelliptic. Let $\tau$ be the hyperelliptic involution of $Y$ and $\phi$ an involution such that $R=Y / \phi$. Then, the quotient of $R^{\prime}=Y / \tau \circ \phi$ is a genus 1 surface. $R^{\prime}$ has a natural representation as $C / \Lambda$, where $\Lambda$ is a lattice. From this, they construct a holomorphic function $f: \mathbf{D} \rightarrow \mathbf{C}$, such that $T_{\Lambda} \circ f$ is a $G$-invariant meromorphic function, where $T_{\Lambda}$ is $\Lambda$-invariant and is used to determine the algebraic equation of the surface.

Explicitly they used $T_{\Lambda}=T_{\tau}$,

$$
T_{\tau}=-\frac{\omega}{K} \prod_{k=0}^{\infty} \frac{\left(\omega-\zeta^{2 k}\right)^{2}\left(1-\zeta^{2 k+2} \omega\right)^{2}}{\left(\omega-\zeta^{2 k+1}\right)^{2}\left(1-\zeta^{2 k+1} \omega\right)^{2}}
$$

where $\zeta=\exp (\pi i \tau), \omega=\exp (\pi i z)$ and

$$
K=4 \prod_{k=0}^{\infty}\left(\frac{1+\zeta^{2 k}}{1+\zeta^{2 k-1}}\right)^{4}
$$

The outline of the rest of this paper is as follows. Section 2 reviews the fundamental concepts about associated graphs to hyperelliptic Riemann surfaces and describes the canonical and explicit cell decomposition of the moduli space of close Riemann of genus two, $M_{2}$. Section 3 shows how to find the graph associated to the well know Bolza's curve as well as its angle coordinates. Section 4 studies the circle pattern uniformization problem for the only singularity of $M_{2}$. On section 6 , we introduce symmetric octagons as a tool to solve the circle pattern uniformization problem for generic graphs of genus two. Finally, on section 7, we will give some conclusions of this work. 


\section{A view of the canonical cell decomposition of $M_{2}$}

The canonical and explicit cell decomposition of the moduli space of closed Riemann surfaces of genus two described on [2] is based on the combinatorial structure of $M_{2}$, the solution of circle pattern realization problems associated to $M_{2}$, and the existence of 6-dimensional polytope complex which can be viewed as a compactification of covering orbifold of $M_{2}$.

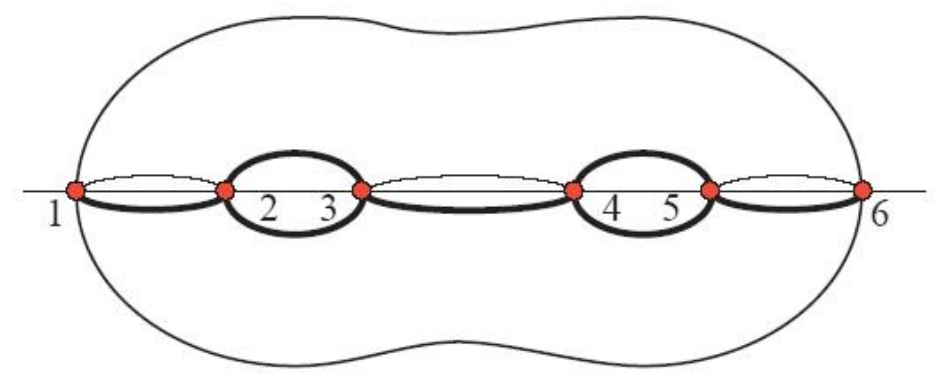

Figure 1: On a closed genus two Riemann surface with its hyperbolic metric, the set of its Weierstrass points determines a graph, the 1-skeleton of its Voronoi diagram that can be projected to the 2 dimensional sphere. Here, we represent with red points the Weierstrass points of a surface which are the 6 fixed points of its hyperelliptic involution.

We can reveal the combinatorial structure of $M_{2}$ by associating a graph to each closed Riemann surface of genus two. Indeed, since each Riemann surface $R$ of genus two has a hyperbolic metric as well as a hyperelliptic involution, we can obtain the Voronoi cell decomposition of $R$ with respect to its set of Weierstrass points and determine a unique geodesic graph $\widehat{G}(R)$ which is the 1-skeleton of this decomposition on the surface. See Figure 6 .

The graph $\widehat{G}(R)$ can be projected to the two dimensional sphere because the hyperelliptic involution, $\tau$, of a Riemann surface of genus two $R$ induces an action on $R$ and a natural projection $\rho: R \rightarrow R / \tau$.

The geodesic graph $G(R)=\rho(\widehat{G}(R))$ provides a 2-cell decomposition of the Riemann sphere (geodesic in the induced hyperbolic structure) having exactly 6 faces, each face containing exactly one projection under $\rho$ of a Weierstrass point. 
If $\alpha$ and $\beta$ denote the number of vertices and the number of edges, then the Euler's formula asserts that $\beta-\alpha=4$. Let us denote by $e_{1}, \ldots e_{\beta}$, the edges of $G(R)$. By construction, each vertex $v$ has geodesic circle $C_{v}$ with centre at $v$ and passing through the points $Q_{j}$ corresponding at the faces incident to $v$. The numbers $\theta^{*}\left(e_{1}\right), \ldots, \theta^{*}\left(e_{\beta}\right) \in(0, \pi)$ satisfy some inequalities [2], determined at vertices, and an equality for each face: if $e_{1}, \ldots e_{i_{k}}$ are the incident edges to the face $f_{i}$, then

$$
\sum_{j=1}^{k}\left(\pi-\theta^{*}\left(e_{i_{j}}\right)\right)=\pi,
$$

as each point $Q_{i}$ has total angle $\pi$. Note that the only places on which we have cone angles are at the points $Q_{1}, \ldots Q_{6}$. Summarizing, we have $\beta$ numbers in $(0, \pi)$ and 6 linear relations between them. See example on Fig 2. Also, the reduced group $A u t^{r e d}(R)=A u t(R) /\langle\tau\rangle$, which is exactly the subgroup of $P S L_{2}(\mathbf{C})$ keeping invariant the set $\left\{Q_{1}, \ldots, Q_{6}\right\}$, keeps invariant $G(R)$, in particular, if two edges of $G(R)$ are equivalent by that group, then they must have the same $\theta^{*}$-value.
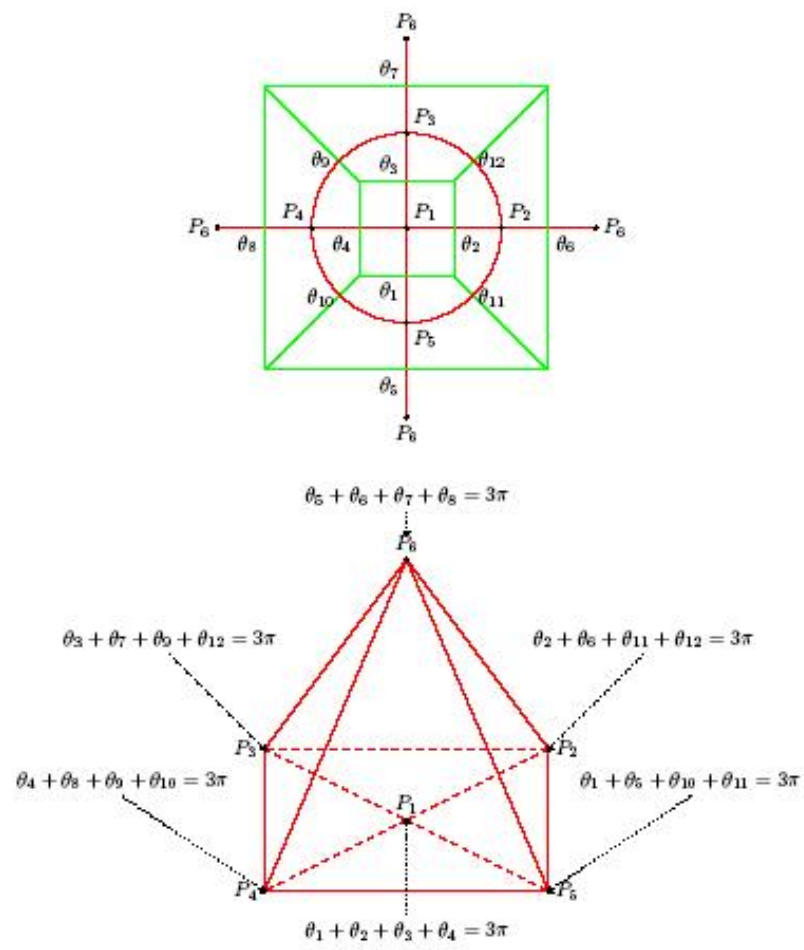
Figure 2: The linear system associated to the generic graph $d_{1}$ has an equation associated to each face of $d_{1}$, the green graph. Equivalently, each of these equations is associated to each vertex of the red graph, dual of $d_{1}$. In addition, we have the constraints $0<\theta_{i}<\pi$ for each $i$.

If $G(R)$ is generic (i.e., all vertices have degree 3), then for $R^{\prime}$ close to $R$ (say that the corresponding values $Q_{j}{ }^{\prime}$ s are near to the values of $Q_{j}^{\prime}$ up to a Möbius transformation and permutation of indices) then $G\left(R^{\prime}\right)$ is isotopic to $G(R)$. By Euler's formula, such generic graph has 12 edges.

The family of graphs associated to closed Riemann surfaces of genus two was computed explicitly in [2]. An important observation for this computation is the fact that two arbitrary graphs of this family are connected by a sequence of contraction or expansion moves, see Figure 3. Then, if we choose any of the graphs on Figure 4, we can generate a family of graphs that includes the associated graphs to Riemann surfaces of genus two. However, this family also includes graphs which are not associated to any Riemann surface of genus two. This leads to the realization problem of determining whether or not a given graph is equivalent to the graph associated to a Riemann surface. On [2] this realization problem was solved by considering an equivalent collection of circle pattern problems, which is described next.

An interesting feature of any graph $G$, associated to a hyperelliptic Riemann surface $R$, is that it has a dual graph $G^{\prime}$. The set of nodes of $G^{\prime}$, $W$, is the set of all the projections of the Weierstrass point of $R$. An edge of $G^{\prime}$ is defined as the geodesic segment joining $W_{i} \in W$ and $W_{j} \in W$ for each common edge of the Voronoi diagram, whose centers are $W_{i}$ and $W_{j}$. From the dual graph $G^{\prime}$, we can also obtain a collection of circles since each face of $G^{\prime}$ has its vertices on a unique circumference whose center is in $W$. the set if vertices of the graph $G$, we call this collection of circumferences the circle pattern associated to $R$.

Circle patterns have been studied by several authors [26, 27, 28, 30], and also by Springborn which in his $\mathrm{PhD}$ thesis [30] proved a result that allow us to determine all possible circle patterns on the hyperbolic sphere that exists for the genus two case, and could be applied more generally to surfaces on the hyperelliptic locus of $M_{g}$. 
$H_{1}$

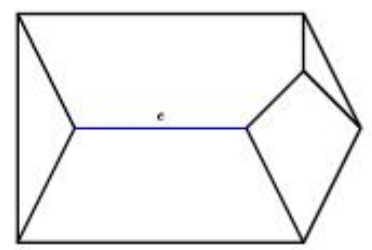

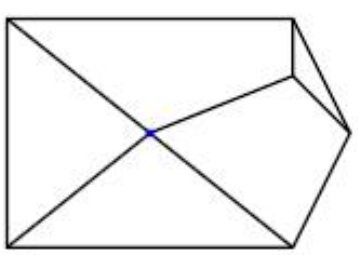

$H_{2}$

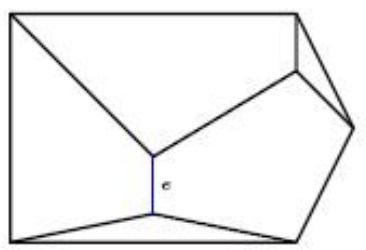

Figure 3: A Whitehead move on the blue edge on graph $H_{1}$, contracts the edge to a point, as shown on the middle graph, followed by an edge expansion as shown on the right graph. The result is the new graph which is represented on the right.

On Figure 4, we describe the combinatorial structure of $M_{2}$ at the generics level, consisting of all cubic graphs associated to a closed Riemann surface of genus two. Two graphs are connected by an edge if they are connected by Whitehead moves.

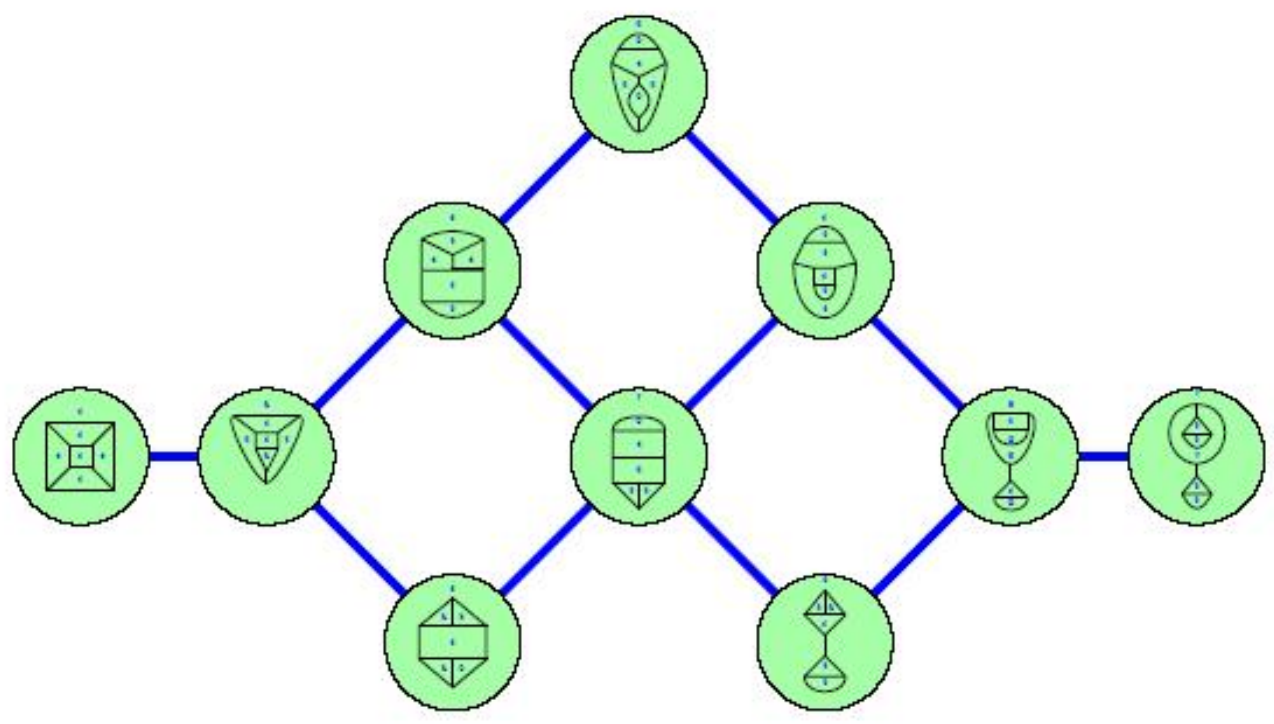

Figure 4: $M_{2}$ combinatorial structure 


\section{Bolza's curve}

One of the most interesting Riemann surfaces of genus two is the Bolza curve, $S_{B}$. Below, we enumerate some known facts about this curve [12]:

1. The projection, $S_{B} / \tau$, of this curve to the Riemann sphere has 6 singular points, the intersections of the Riemann sphere with the coordinates axes

2. an algebraic equation of $S_{B}$ is $y^{2}=x^{5}-x$

3. $S_{B}$ can be represented as $\mathbf{H} / \Gamma$, where $\Gamma$ is a normal subgroup of an appropriately oriented triangle group $T^{\star}$

4. the automorphism group of $S_{B}$ is $T^{\star} / \Gamma$

5. $S_{B}$ can be identified by a cyclotomic construction. In this construction, the lattice in $\mathbf{C}$ is a ring of cyclotomic integers with trace (sum of conjugates) norm

6. $S_{B}$ has $12 \pi_{1}$-systoles and $12 H_{1}$-systoles. In addition, the function $p$ : $\pi_{1}\left(S_{b}\right) \rightarrow H_{1}\left(S_{b}\right)$ maps the $\pi_{1}$ systoles one-to-one to the $H_{1}$ systoles

7. $S_{B}$ is an extremal surface with $N_{2}=N\left(S_{B}\right)=\sqrt{2}$

8. the $\pi_{1}$-systoles of $S_{B}$ are the curves on Figure 7 . Hence, they belong to the set of sides of the Delaunay triangulation associated to the fixed point of the hyperelliptic involution of $S_{B}$

9. $l_{2}=l\left(S_{B}\right)+2 \operatorname{arcosh}(1+\sqrt{2})$. Therefore, $l$ and $N_{J}$ take a maximum at $S_{B}$.

By part 1 of the above properties, we can say that the associated graph of $S_{B}$ is the generic graph $d_{1}$ and also it has angle coordinates $\left(\frac{3 \pi}{4}, \frac{3 \pi}{4}, \frac{3 \pi}{4}, \frac{3 \pi}{4}, \frac{3 \pi}{4}, \frac{3 \pi}{4}, \frac{3 \pi}{4}, \frac{3 \pi}{4}, \frac{3 \pi}{4}, \frac{3 \pi}{4}, \frac{3 \pi}{4}, \frac{3 \pi}{4}\right)$.

\section{4. $M_{2}$ singularity}

The moduli space of closed Riemann surface of genus two has the structure of a 6 dimensional manifold around all its points except at the projective algebraic curve $R_{P}$ with algebraic equation $y^{2}=x^{5}-1$. To identify $R_{P}$, we consider the description given in [12], where $R_{P}$ is described as the double cover of the Riemann sphere with $W=\left\{e_{5}, e_{5}^{2}, \ldots, e_{5}^{5}=1, \infty\right\}$ as 
Weierstrass set. By opening the Riemann sphere along the geodesic connecting $\infty$ with $e_{5}, e_{5}^{2}, \ldots, e_{5}^{5}$, we obtain a regular $\frac{\pi}{5}$ hyperbolic pentagon. By the symmetry of this pentagon, we can say that the Voronoi diagram associated to $R_{P}$ is the green graph on Figure 5. An interesting feature of this graph is that it coincides with its dual, in black. To compute the exterior angles associated to this graph, note that $\theta_{1}=\theta_{2}=\ldots,=\theta_{5}$ and $\theta_{6}=\theta_{6}=\ldots=\theta_{10}$. In addition, as the cone angle at the center of the circle is $\pi$, we have $5\left(\pi-\theta_{1}\right)=\pi$ and at any other vertex $2\left(\pi-\theta_{6}\right)+\left(\pi-\theta_{1}\right)=\pi$. Therefore, $\theta_{1}=\theta_{2}=\ldots,=\theta_{5}=\frac{4 \pi}{5}$ and $\theta_{6}=\theta_{6}=\ldots=\theta_{10}=\frac{3 \pi}{5}$.

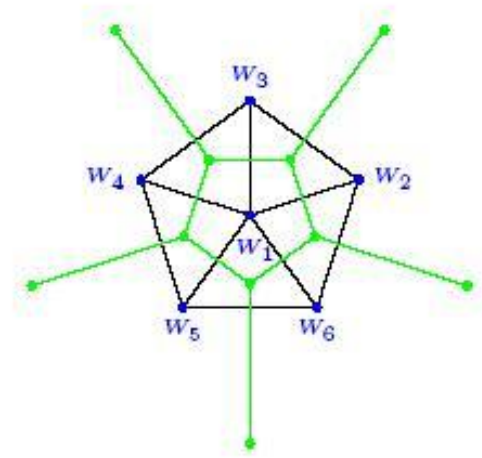

Figure 5: The graph associated to the surface $y^{2}=x^{5}-1$ (in green) and its dual graph (in black). In this representation, we should assume that the exterior nodes in green are identical.

\section{The curve $y^{2}=x^{6}-1$}

In general, a real hyperelliptic curve is a curve of some genus $g$ that can be defined by an equation of the form $y^{2}=P(x)$, where $P(x)$ is a real polynomial and the complex conjugation induces an anti-holomorphic involution which keeps $g+1$ closed curves fixed. If this is the case, $P(x)$ can be chosen with $2 g+2$ distinct real roots. In the case of genus two, its equation has the form $y^{2}=\left(x-\lambda_{1}\right)\left(x-\lambda_{2}\right) \ldots\left(x-\lambda_{6}\right)$, where $\lambda_{1}, \lambda_{2}, \ldots, \lambda_{6}$ are real numbers. In the special case where the inverse image of the projection of $\lambda_{i}, i=1,2, \ldots, n$ in the sphere are equidistant, the associated $C E$-graph for the corresponding Riemann surfaces is the spacial graph which has only two vertices. The algebraic equation of the surface is

$$
y^{2}=(x-1)(x+1)(x-2-\sqrt{3})(x+2+\sqrt{3}(x-2+\sqrt{3})(x+2-\sqrt{3})
$$


Another way to get the same curve is to take as fixed point of the curve the 6th-roots of unity, because this representation of the marked sphere can be obtained from the above description by a three dimensional rotation. Hence, $y^{2}=x^{6}-1$ is another equation of the curve [12].

\section{Symmetric octagons for generic graphs}

The goal of this section is to show how we can construct an octagon representation $\operatorname{Octo}(R)$ of a Riemann surface of genus two, $R$, which is associated to a generic graph $G$ with an angle coordinates $\Theta$.

It is known that each Riemann surface of genus two has an admissible octagon representation [1] which satisfies the following properties:

1. It is centered in the origin (in the disk model)

2. the Weierstrass fixed points of $R$ are: the origin, the middle points of the sides and the vertices of $\operatorname{Octo}(R)$

3. each side of the octagon is identified with its opposite.

Our goal is to build $\operatorname{Octo}(R)$ from the knowledge of $G$ and its angle coordinates $\Theta$.

To describe our construction, define $H$ to be the dual graph of $G$ viewed as an embedded graph on $R$ which is $\mathbf{S}^{2}$, the Riemann sphere with the hyperbolic metric, where the vertices of $H$ correspond to the six $\frac{\pi}{2}$ cone points which are the projections of the Weierstrass points of $R$.

We can represent $H$ as a hyperbolic quadrangle $Q$ : If a vertex $P_{1}$ of $H$ has 4 neighbouring vertices $P_{2}, P_{3}, P_{4}, P_{5}$, with $P_{i} \neq P_{j}$ for $i \neq j$, we can make cuts from $P_{1}$ to $P_{j}$, where $j=2,3,4,5$, and open up. Observe that a copy of $P_{1}$ is at each of the four vertices of $Q$ and $P_{i}$, where $i=2,3,4,5$, is the middle point of each side of $Q$ and subdivide it into two segments that should be identified. The angle at these segment middle point is $\pi$, so we really get a planar hyperbolic quadrangle. In addition, on the interior of $Q$, we can find $P_{6}$, the sixth triangulation vertex, see Figure 6 . 

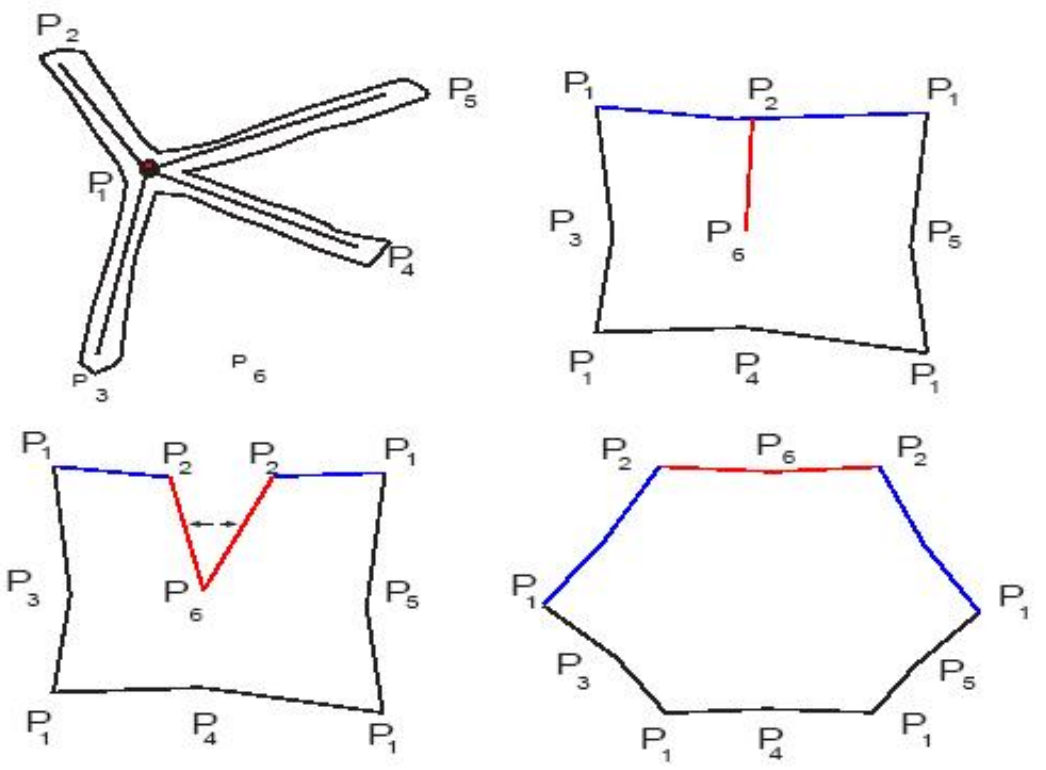

Figure 6: To construct a hexagon representation of a two dimensional hyperbolic sphere with 6 marked points, first we choose a point $P_{1}$ and proceed with 4 cuts as indicated on the first row. Then by cutting and opening along the red line, we will have the desired hexagon representation as illustrated on the second row.

We will obtain $R$ as the double covering space of the sphere, coming from the gluing of two pair of pants $\underline{R}$ and $\underline{R}$, each of which will have a hexagon representation coming from $Q$.

Observe that to glue $\underline{R}$ and $\underline{R^{\prime}}$ to get $R$, we need to choose 3 disjoint geodesics, $\gamma_{1}, \gamma_{2}, \gamma_{3}$ in $\underline{R}$, and corresponding geodesics, $\gamma_{1}{ }^{\prime}, \gamma_{2}{ }^{\prime}, \gamma_{3}{ }^{\prime}$ in $\underline{R^{\prime}}$, joining the projections of the Weierstrass points of $R . \gamma_{i}$ is divided into two parts, $a_{i}$ and $b_{i}$, by the Weierstrass point projection of $R$ in $\underline{R}$. Similarly, the corresponding geodesic $\gamma_{i}^{\prime}$ is divided into $a_{i}^{\prime}$ and $b_{i}^{\prime}$ by the Weierstrass point projection of $R$ in $\underline{R}^{\prime}$. Hence, the segment $a_{i}$ should be glued to $b_{i}^{\prime}$ and also $b_{i}$ glued to $a_{i}^{\prime}$.

We will follow the following procedure to get a symmetric octagon associated to the triangulation determined by the generic graphs $d_{i}$, for $i=1,2,3,4,5,6,7,9,10$. 
A. Choose 4 geodesic segments starting at vertex $P_{1}$ with extremes $P_{2}$, $P_{3}, P_{4}, P_{5}$ and build a basic quadrangle $(Q)$

B. take 2 copies of quadrangles and build 2 hexagon representations of the surface projection as described on Figure 6

C. build 2 hexagons representation whose union is the surface $\left(\underline{R}, \underline{R^{\prime}}\right)$.

D. build an octagon representation of the surface by rotating and gluing one hexagon to the other along one of its common sides.

We might need to interchange some regions (on red) to get $R$, because we need to choose 3 geodesics that join different pair of pants. Then, we need to check that the crossings of these geodesics lead to the right pair of pants copy.

In the sequel, we describe symmetric octagon representations for all generic graphs $d_{i}, i=1,2, \ldots, 10$. Each of our octagons representation defines a triangulation whose dual is $d_{i}$. In addition from our representations, it is possible to get the Fenchel Nielsen coordinates of the respective surface, and one approximation of its algebraic equation.

On Figures 7-16, we label the edges of the triangulation in the first hexagon with the numbers $i=1,2, \ldots, 12$ and the corresponding ones in the second hexagon with $\underline{\underline{1}}$. In addition, the edges $i$ and $\hat{i}$ are identified as well as $i$ and $\underline{\hat{\imath}}$

By changing the pair of pants coordinates of a Riemann surface $R$ of genus 2, we expect to see a continuous deformation of the graph associated to $R$ and able to carry a given marking of $R$ to its neighbors. 
A.
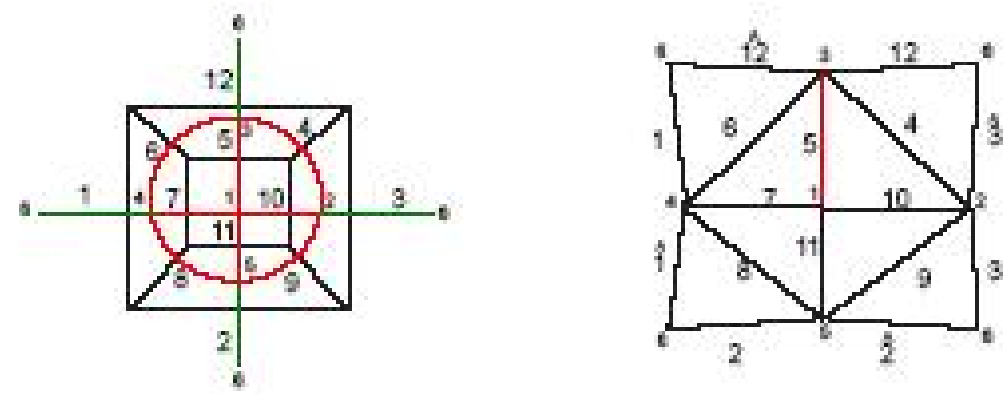

B.
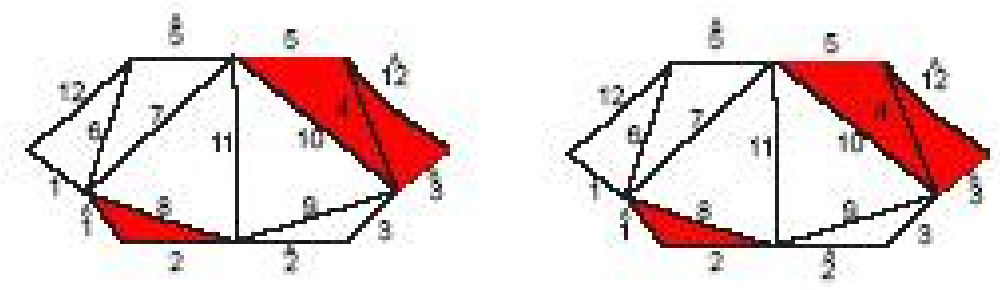

C.
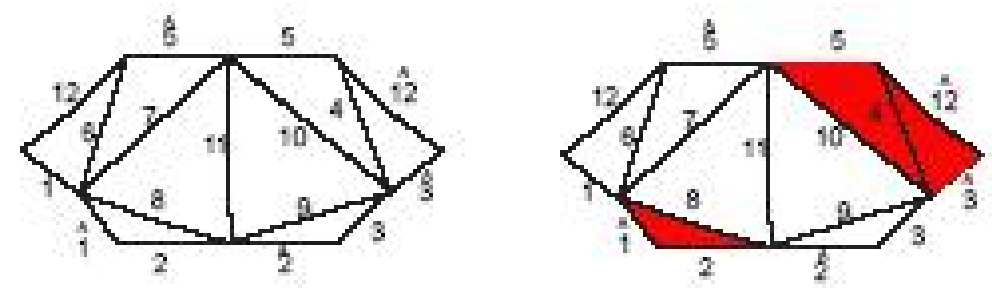

D.

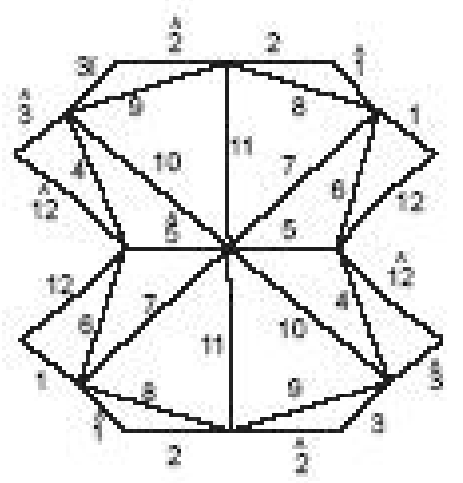

Figure 7: Condructicn of an octagon representation for $d_{1}$. 
A.
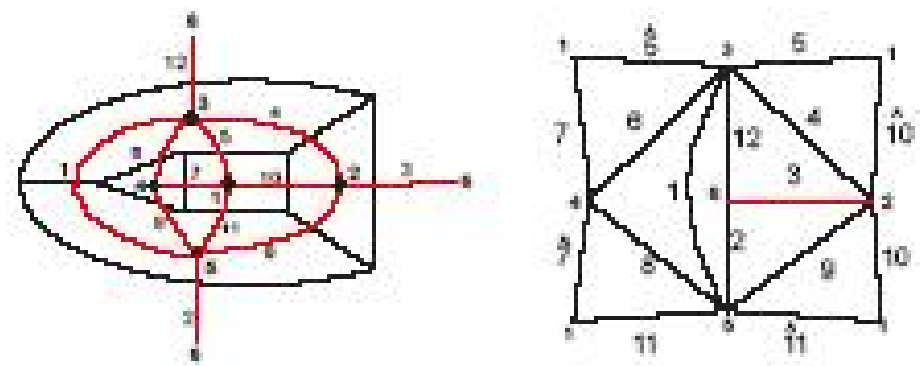

B.
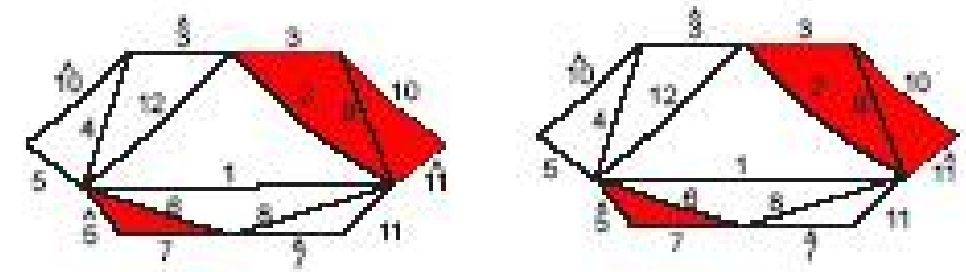

G.
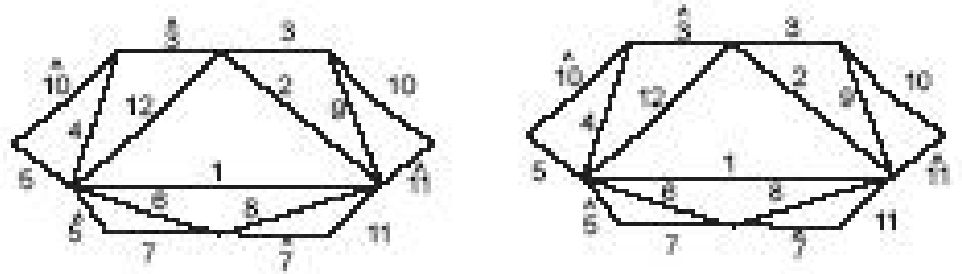

D.

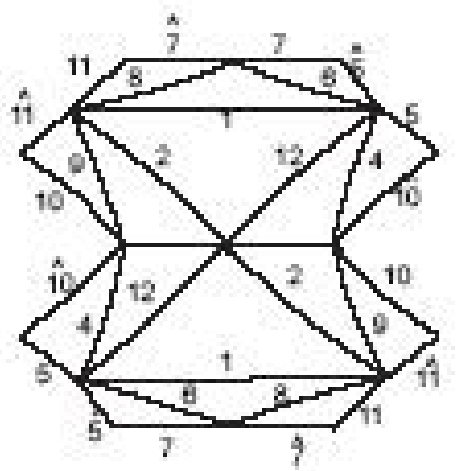

Ftgue 8: Condruction of an otagon repesentation for $d_{2}$ 
4
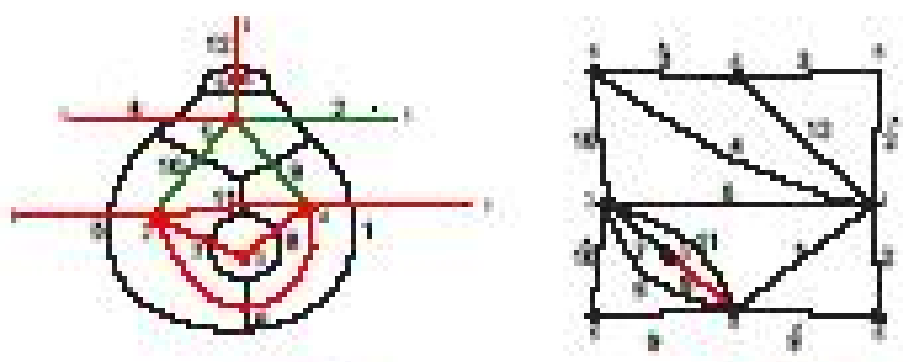

E.
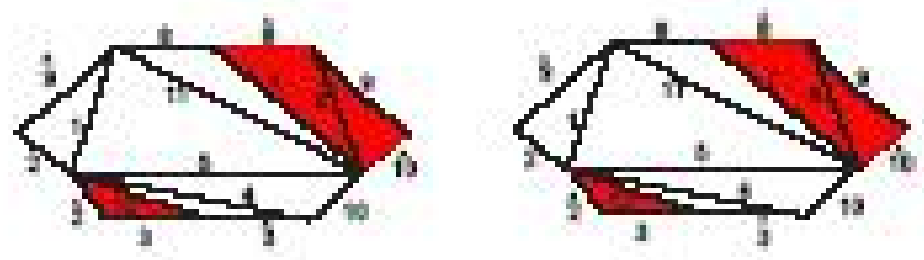

C.
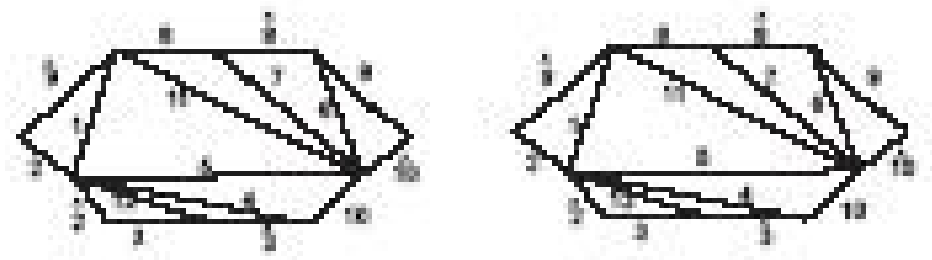

E.

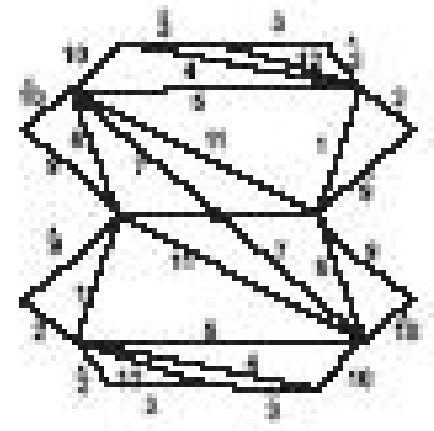

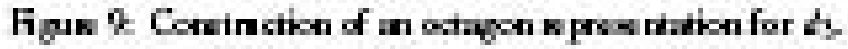


A.
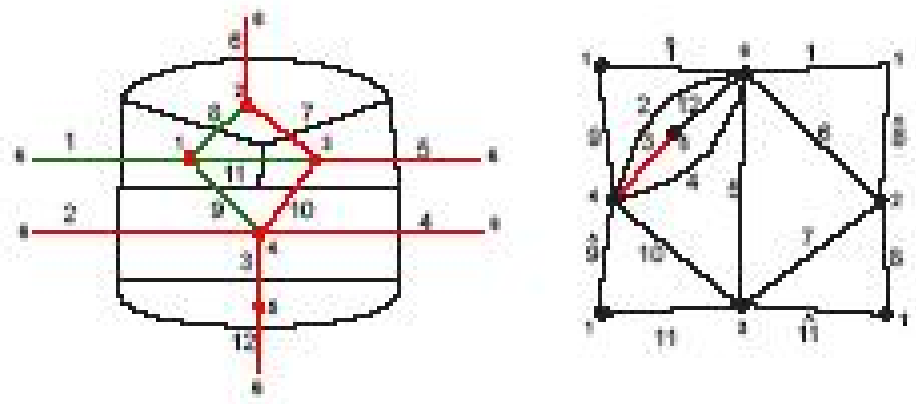

B.
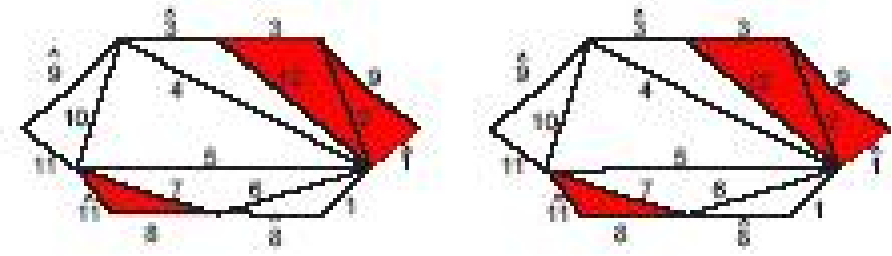

C.
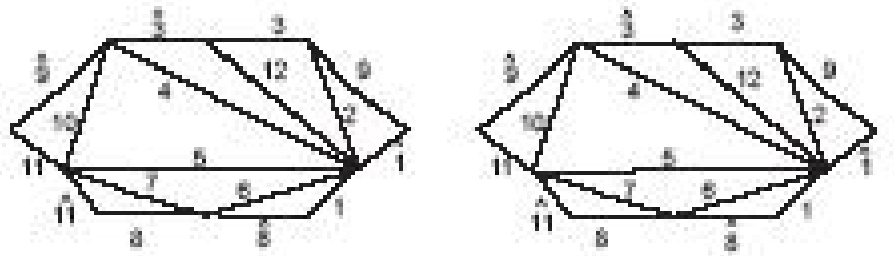

D.

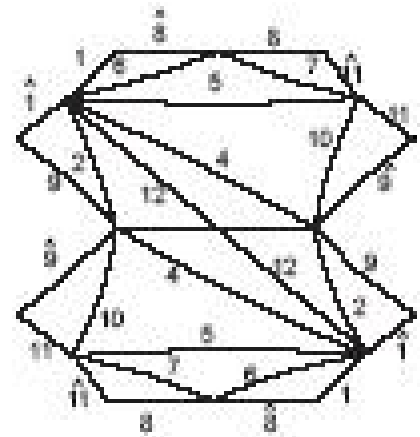

Figue 10: Contruction of an octagon eppesentation for $d_{4}$ 
A.
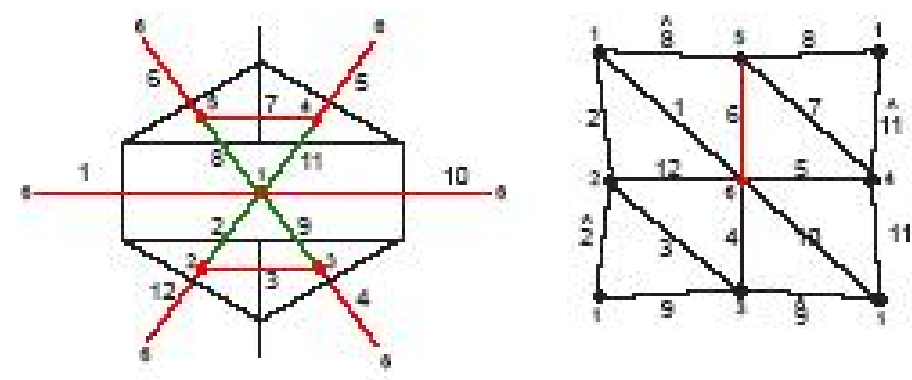

B.
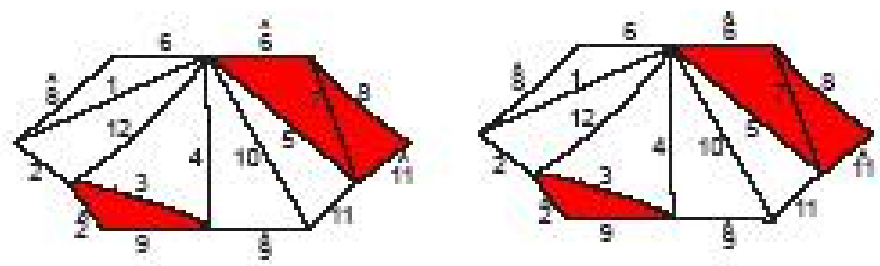

c.
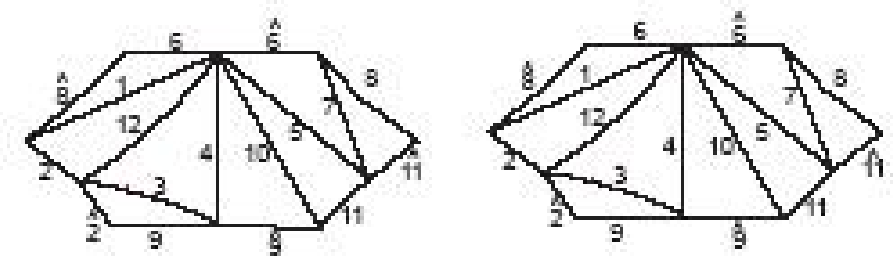

D.

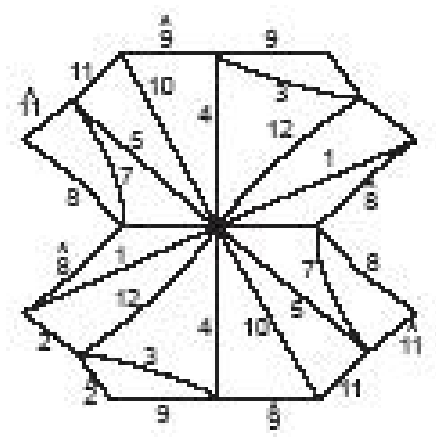

Figure 11: Contruction of an octagon wpresentarion for ds. 
A.
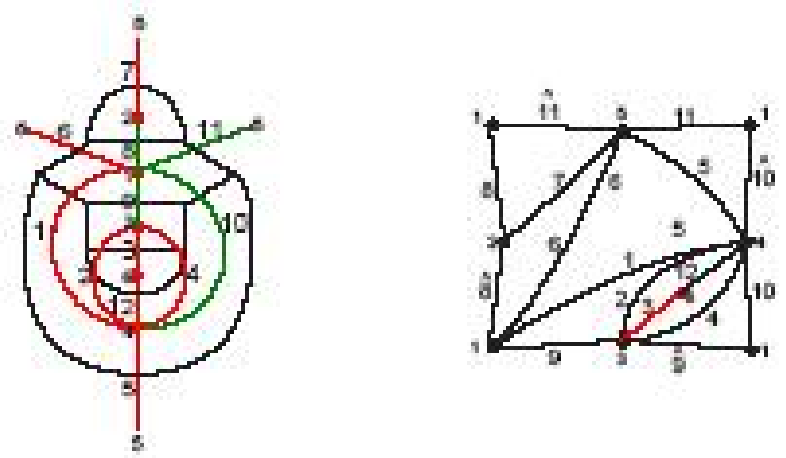

B.

C.
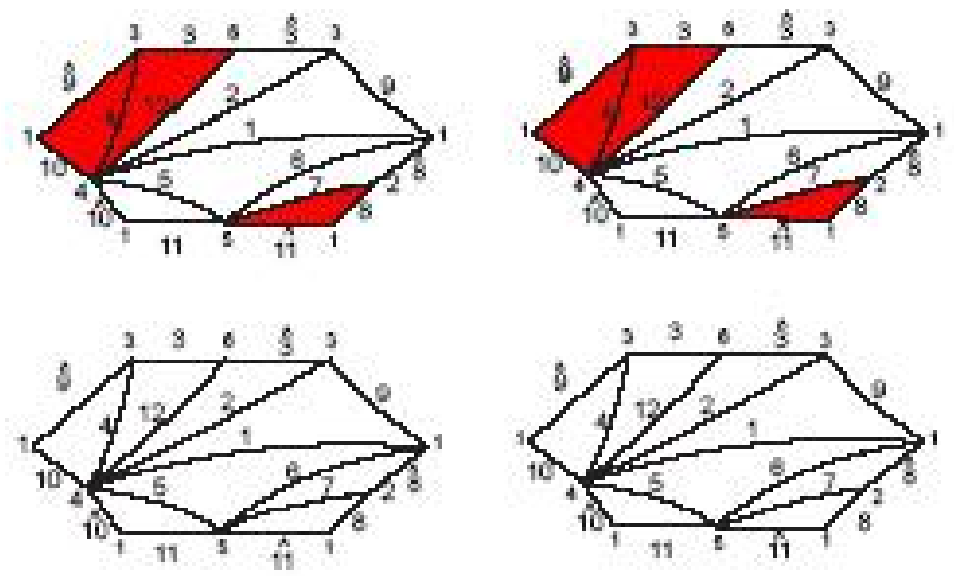

D.

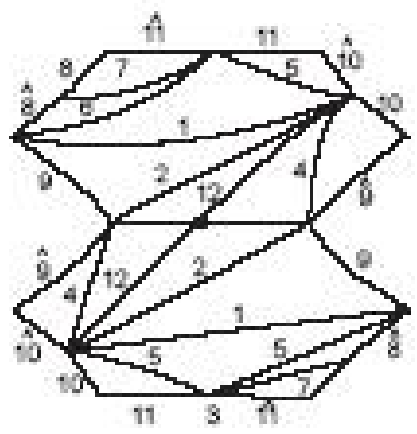

Figure 12: Construction of an octagon re presentation for $d_{s}$. 
A.
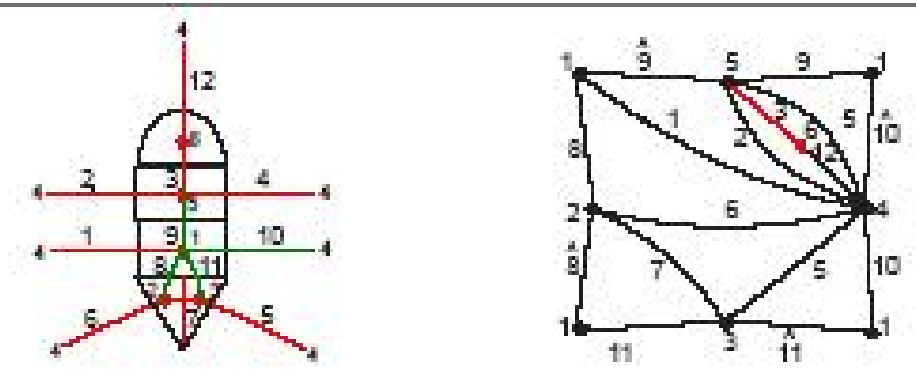

B.
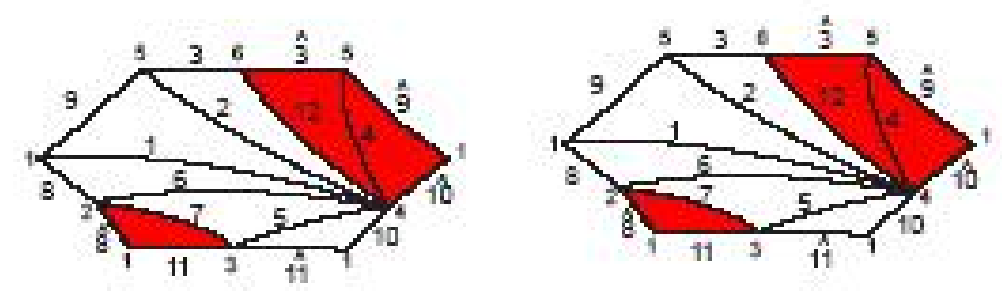

C.
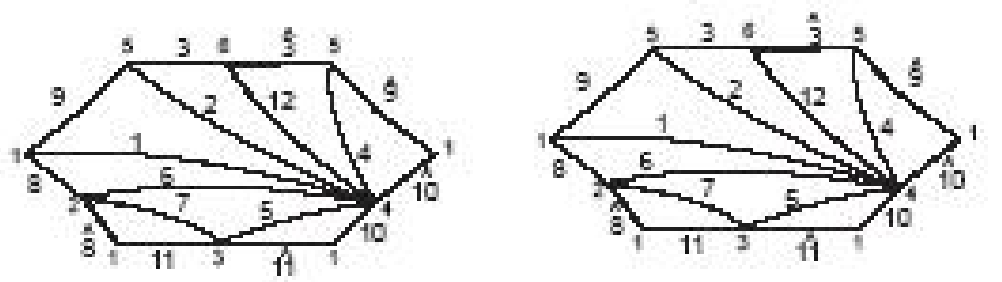

D.

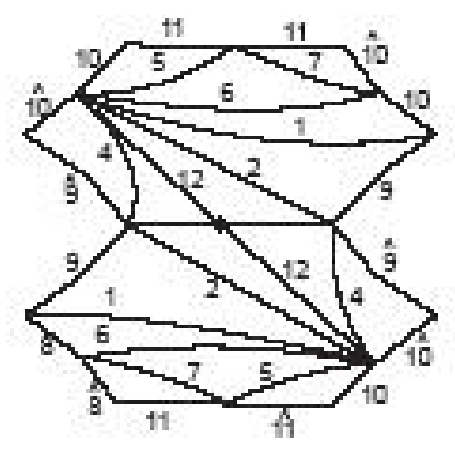

Figure 13: Construction of an octagon re presentation for $d_{7}$. 

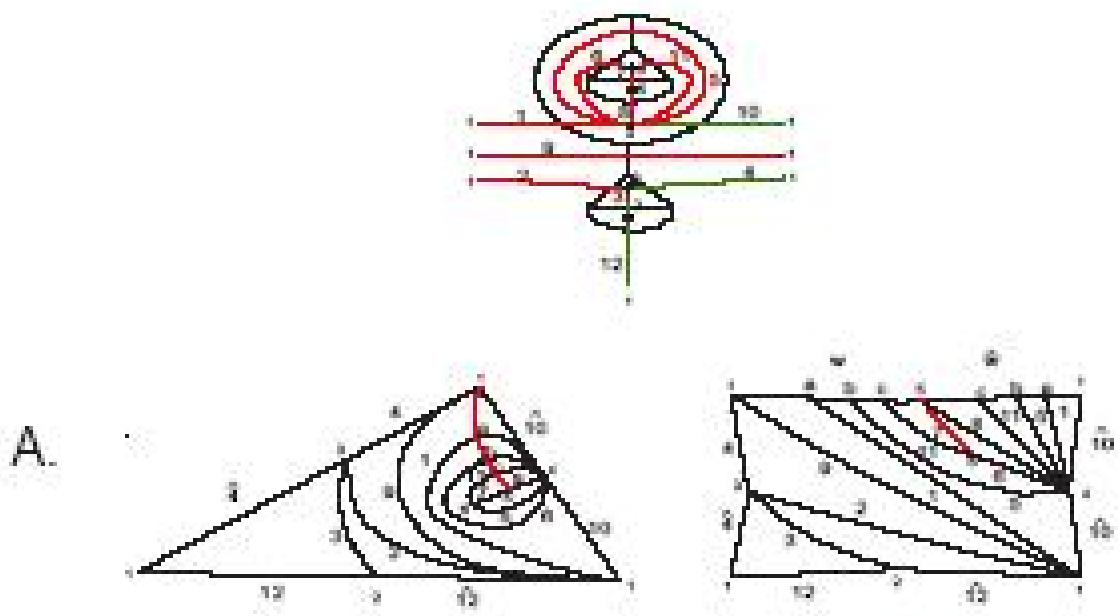

B.
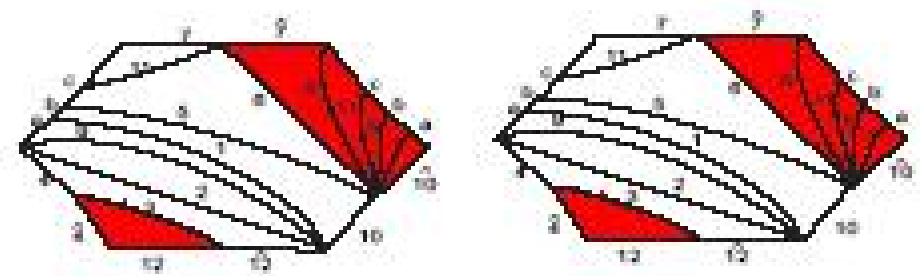

C.
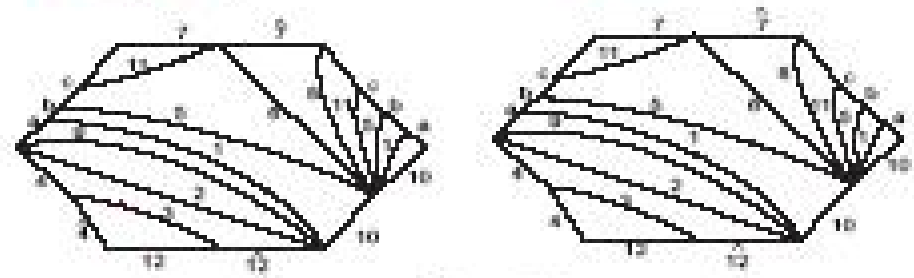

D.

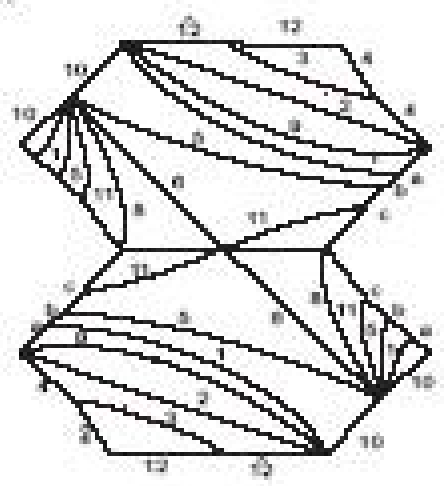

Figue 14: Construction of an octagon representation for d 
A.
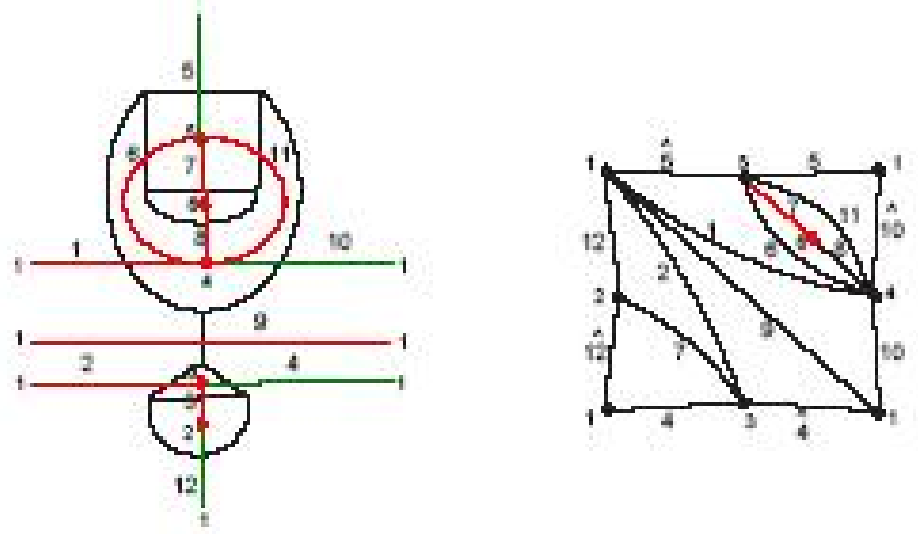

B.
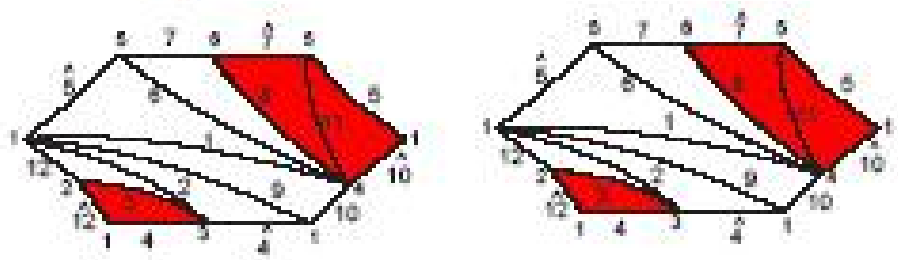

C.
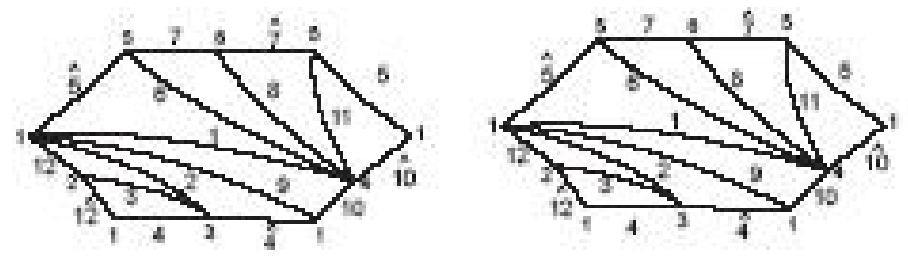

D.

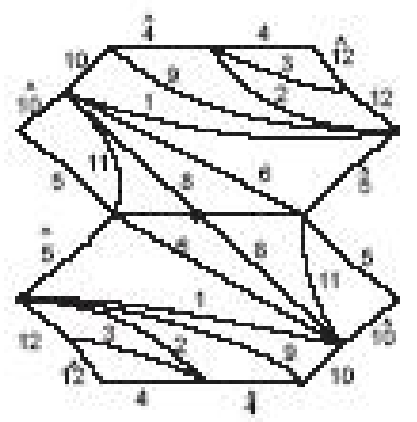

Figure 15: Construction of an octagon representation for $d$. 
A.
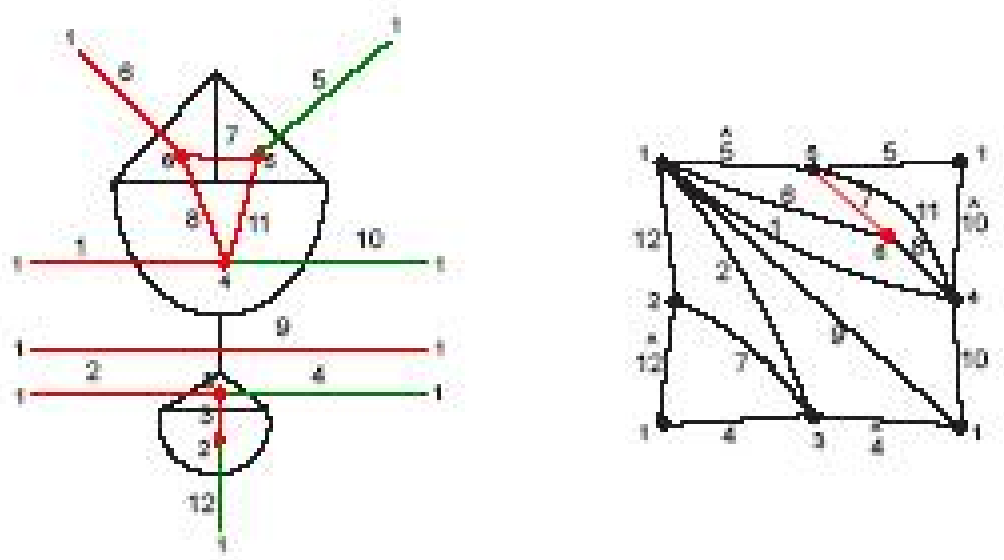

$B$.
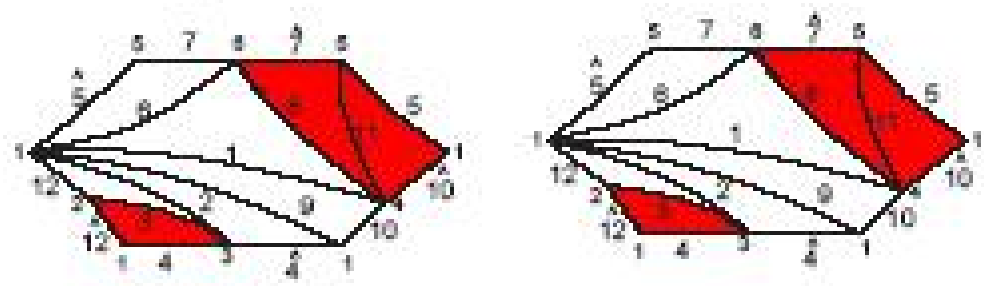

C.
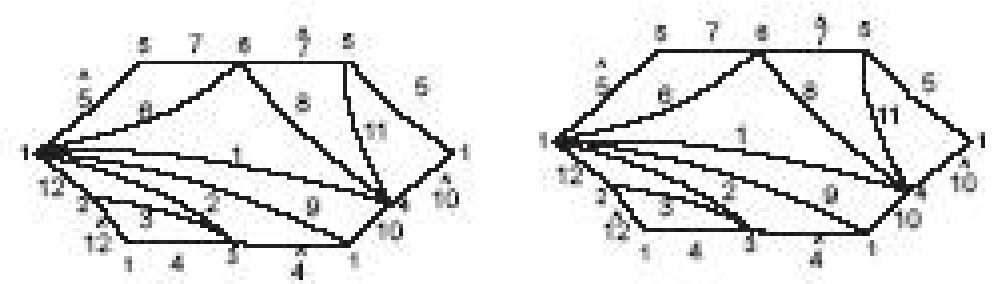

D.

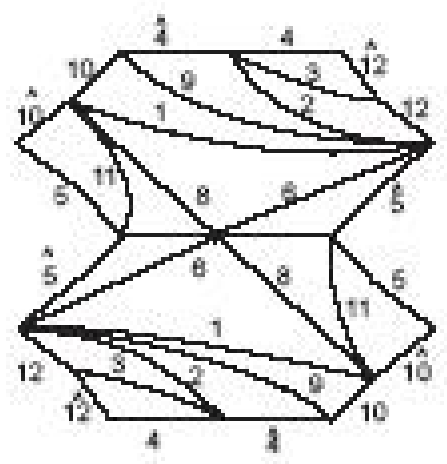

Figure $1 \propto$ Construction of an cotagon repreantation for $d_{10}$. 


\section{Conclusion}

The circle pattern uniformization problem for Riemann which we have introduced is a new challenge that aims to integrate three of the most important perspectives of the concept of Riemann surface and show the structure of Riemann surfaces while gaining an intuitive understanding by using graphical representations. Solving the circle pattern uniformization problem would reveal how Riemann surfaces are interconnected, and add a dynamical view to this subject because paths on the moduli space of closed Riemann surfaces could be understood as single surfaces which are being transformed, or as hyperbolic cone spheres whose edges are being transformed by contractions or expansions moves.

We did not implement the numerical approach to compute equations of Riemann surfaces of genus two described on this paper.

The dynamical view of Riemann surfaces is attractive for potential applications. For example in neurosciences, many degenerative diseases change the human brain's cortex which we can model as a surface which changes through time. Then in this setting, the challenge is to understand how the structural changes that the brain cortex suffer as a mathematical surface mirror the evolution of medical conditions. A sophisticated mathematical understanding of Riemann surfaces could lead to medical advances. In particular but not restricted to research on Alzheimer and HIV diseases since these diseases impact brain's structures and the morphology of human cortex.

\section{References}

[1] A. Aigon: Transformation Hyperboliques et Courbes Algébriques en genre 2 et 3, UNIVERSITÉ MONTPELLIER II, thèse, (2001).

[2] A. J. R. Rodado: Weierstrass Points and Canonical Cell Decompositions of the Moduli and Teichmüller Spaces of Riemann Surfaces of Genus Two, University of Melbourne, PhD thesis, http://repository.unimelb.edu.au/10187/2259

[3] A. J. R. Amaris, M.P. Cox: A Flexible Theoretical Representation of The Temporal Dynamics of Structured Populations as Paths on Polytope Complexes, Journal of Mathematical Biology, 2014, DOI 10.1007/s00285-014-0841-4 
[4] AMPL, A Modeling Language for Mathematical Programing, http://www.ampl.com

[5] P. Buser: Geometry and Spectra of Compact Riemann Surfaces, Progress in Mathematics, Vol 106, Birkhauser, (1992).

[6] Bowditch B. and B. Epstein: Natural Triangulations Associated to a Surface, Topology vol 27, No 1, pp. 91-117, (1988).

[7] P. Buser and R. Silhol: Some remarks on the uniformization function in genus 2, EPFL Lausanne, Université Montpellier II, http://www.math.univ-montp2.fr/ rs/genus2unif.pdf, 2005.

[8] M. Franz: Convex: a Maple package for convex geometry http://wwwfourier.ujf-grenoble.fr/ franz/convex/.

[9] H. M. Farkas and I. Kra : Riemann Surfaces, Graduate Texts in Mathematics 71, Springer-Verlag: New York, Heidelberg, Berlin (1991).

[10] D. Griffiths: The Side Pairing Elements of Maskit's Fundamental Domain for the Modular Group in Genus Two, Annales Academiae Scientiarum Fennicae Mathematica, Vol 26, pp. 3-50, (2001).

[11] D. Griffiths: At most 27 length inequalities define Maskit's fundamental domain for the modular group in genus two, Geometry and Topology Monographs, vol 1, The Epstein Birthday Schrift, pp. 167-180, pp. 3-50, (1998). th.-Phys. Kl. 88, 141-164, 1936.

[12] T. Kuusalo and M. Näätänen: Geometric Uniformization in Genus 2, Annales Academiae Scientiarum Fennicae, Series A.I. Mathematica vol 20, pp. 401-418, (1995).

[13] T. Kuusalo and M. Näätänen: Weierstrass points of extremal surfaces in genus 2, http://www.math.jyu.fi/research/pspdf/231.pdf

[14] J. Harris and I. Morrison:Moduli of Curves, Graduate Texts in Mathematics, Springer-Verlag, (1998).

[15] A. Hass and P. Susskind: The Geometry of the Hyperelliptic Involutions in Genus Two, Proceeding of the American Mathematical Society, vol 105, 1, January (1989).

[16] T. Jorgensen and M. Naatanen: Surfaces of Genus 2: Generic Fundamental Polygons, Quart. J. Math. Oxford (2), 33, pp. 451-461, (1982). 
[17] J. D. McCarthy: Weierstrass points and $\mathbf{Z}^{2}$ homology, Topology and its Applications 63, pp. 173-188, (1995).

[18] G. Mcshane: Weierstrass Points and Simple Geodesics, Bull. London Math. Soc. 36, pp. 181-187, (2004).

[19] B. Maskit: New Parameters for Fuchsian Groups of Genus 2, Proceeding of The American Mathematical Society, vol 127, No 12, pp. 3643-3652, (1992).

[20] B. Maskit: Matrices for Fenchel-Nielsen Coordinates, Annales Academiae Scientiarum Fennicae, Mathematica vol 26, pp. 267-304, (2001).

[21] B. Maskit: A Picture of the Moduli Space, Invent. Math. 126 , pp. 341-390, (1996).

[22] J. Milnor: Hyperbolic Geometry: The First 150 Years, Bulletin of The American Mathematical Society, Vol. 6, No 1, January (1982).

[23] M. Näätänen: On the Stability of Identification Patterns for Dirichlet Regions, Annales Academiae Scientiarum Fennicae, Series A.I. Mathematica vol 10, pp. 411-417, (1985).

[24] M. Näätänen: A Cellular Parametrization for Closed Surfaces with Distinguished Point, Annales Academiae Scientiarum Fennicae, Series A.I. Mathematica vol 18, pp. 46-64, (1993).

[25] A. Rodado Amaris and G. Lusares: Parametrised databases of surfaces based on Teichmüller theory, CUBO A Mathematical Journal, Vol.18, No 01, pp. 69-88. December (2016).

[26] I. Rivin: Combinatorial optimization in geometry, Adv. in Applied Math. 31:1, pp. 242-271, (2003).

[27] I. Rivin and C. D. Hodgson: A characterization of compact convex polyhedra in hyperbolic 3-space, Invent. Math., 111 : pp. 77-111, (1993).

[28] B. Rodin and D. Sullivan: The convergence of circle packing to the Riemann mapping, J. Differential Geometry, 26, pp. 349-360, (1987).

[29] K. D. Semmler and M. Seppälä: Numerical Uniformization of Hyperelliptic curves, Proc. ISSAC, (1995). 
[30] B. Springborn: Variational Principles for Circles Packing, Ph.D. thesis, arXiv:math.GT/031236 v.1, 18 Dec 2003.

\section{Armando Rodado Amaris}

Departamento de Ciencias Exactas

Universidad de los Lagos

Av. Fuchslocher 1035,

Osorno,

Chile

e-mail : armando.rodado@ulagos.cl

and

\section{Gina Lusares}

Universidad del Valparaiso

Blanco 951,

Valparaiso,

Chile

e-mail : gina.lusares@postgrado.uv.cl 